\title{
Optimized Route Network Graph as Map Reference for Autonomous Cars Operating on German Autobahn
}

\author{
Paul Czerwionka, Miao Wang \\ Artificial Intelligence Group \\ Institute of Computer Science \\ Freie Universität Berlin, Germany
}

\begin{abstract}
This paper describes several optimization techniques used to create an adequate route network graph for autonomous cars as a map reference for driving on German autobahn or similar highway tracks. We have taken the Route Network Definition File Format (RNDF) specified by DARPA and identified multiple flaws of the RNDF for creating digital maps for autonomous vehicles. Thus, we introduce various enhancements to it to form a digital map graph called RNDFGraph, which is well suited to map almost any urban transportation infrastructure. We will also outline and show results of fast optimizations to reduce the graph size. The RNDFGraph has been used for path-planning and trajectory evaluation by the behavior module of our two autonomous cars "Spirit of Berlin" and "MadeInGermany". We have especially tuned the graph to map structured high speed environments such as autobahns where we have tested autonomously hundreds of kilometers under real traffic conditions.
\end{abstract}

\section{INTRODUCTION}

Research on autonomous driving has received a major interest in the past few years as they present a great opportunity to improve safety and mobility. It is common that autonomous behavior for cars will first be introduced as passive advanced driver assistance systems (ADAS), such as lane departure warnings and later upgraded to active systems like lane keeping assistants [1]. It is the ultimate aim to have a completely autonomous car that can drive in daily traffic by its own without human intervention. Most autonomous cars are designed to follow a simple perception, decision and action pattern. The system first gathers sensor information such as position, speed, nearby obstacles perceived by different sensors, etc. to fusion them into a environmental model. Based on this model and a given mission a decision is made and then executed.

One important data source that is mostly provided $a$ priori is a GPS-based digital map of the area including road structure, drivable areas, traffic signs, and mission points. Once this map is designed an autonomous car can use its own GPS position to station itself relative to the map. Although local GPS offset can then be corrected a posteriori either by using differential GPS correction and/or camera-based lane keeping assistants, the initial accuracy of the digital map and its road structure is essential for navigation. Moreover, a detailed map computed before-hand can mitigate the need for local correction with high computing cost afterwards. This is especially true for highways and high-speed courses as mapping errors are more crucial when driving at high speeds, performing lane changes or overtaking maneuvers.

An universal format to define such digital road maps has been issued by the Defense Advanced Research Projects Agency (DARPA) for its autonomous vehicle competitions in desert terrain in 2004 and 2005, and in urban environments in 2007. The Grand Challenges 2004 and 2005 used a route definition data format (RDDF) which consists of a list of longitudes, latitudes, speed limits, and corridor widths that define the course boundary. It was later updated to a route network definition file (RNDF) for the Urban Challenge including stop signs, parking spots and intersections.

Although the RNDF specification is fairly easy to understand and sufficient enough to map most road networks, it misses several features and reveals flaws when designing specific road characteristics. In this paper, we will identify these flaws and present a few enhancements to the RNDF which we call RNDFGraph. Furthermore, we will outline few fast optimization steps to reduce graph size and present generated exemplary route networks. The networks are used by our two autonomous cars "Spirit of Berlin" and "MadeInGermany" during experiments on various maps including an German autobahn course which we drove autonomously under real traffic conditions.

The remainder of this paper is structured as follows. In Section II, we give information on the background of autonomous cars in the DARPA Challenge and also describe the architecture of "Spirit of Berlin" and "MadeInGermany". In the following Section III we describe and identify deficiencies of the RNDF. Thereafter, we will present our enhancements as RNDFGraph in Section IV. Section V gives a description on several designed route networks and optimization results. Finally, Section VI presents our conclusions.

\section{RELATED WORK}

\section{A. DARPA Challenges}

The DARPA has organized three challenges for unmanned land vehicles in 2004, 2005 and 2007 [2] [3].

In 2004, the goal was to drive a predefined route of 150 miles within 10 hours in Barstow, California. Over 100 teams participated in this challenge, but none of them managed to complete the whole distance to win. The best performance was accomplished by Carnegie Mellon Red Team's robot 
Sandstorm with 7.36 miles before hitting a road obstacle [4].

DARPA repeated the same challenge in 2005. All but one of the finalists surpassed the distance of Sandstorm in the preceding year. Five vehicles successfully completed the race with the winner robot Stanley from Stanford Racing Team that finished the course in under 7 hours [5].

In 2007, DARPA moved to a urban scenario. The DARPA Urban Challenge (DUC) took place at the site of the nowclosed George Air Force Base in Victorville, California. The goal involved finishing a 60 mile course in urban area in less than 6 hours including obeying all traffic laws while negotiating with other traffic participants and obstacles and merging into traffic. The winner was Tartan Racing (Carnegie Mellon University and General Motors Corporation) with their vehicle Boss and a finishing time of 4 hours and 10 minutes [6].

While the 2004 and 2005 events were more physically challenging for the vehicles, because the robots only needed to operate in isolation with focus on structured situations such as highway driving, the 2007 challenge required engineers to build robots able obey all traffic regulations and make intelligent decisions in real time based on the current situation.

\section{B. Highway Driving}

A couple of dedicated research on autonomous driving on highway environments has been done in the 1990's with an focus of improved safety for high-speed environments. Foremost sponsored by the European research and development funding and coordination organization EUREKA, the PROMETHEUS program (Program for a European Traffic with Highest Efficiency and Unprecedented Safety) was one of the largest research and development project for autonomous vehicles. It produced the VITA robots which drove more than 1000 kilometers on a Paris multi-lane highway in heavy traffic at speeds up to $130 \mathrm{~km} / \mathrm{h}$ [7]. Few years later, the VaMP vehicle by Universität der Bundeswehr München achieved speeds exceeding $175 \mathrm{~km} / \mathrm{h}$ on the German autobahn in a long-distance run from Southern Germany to Denmark in 1995 [8]. Obstacle detection and positioning was computed locally with vision and without GPS assistance. Simultaneously, the CMU-Navlab project in the USA [9] and the PVS-Project in Japan [10] initiated similar research projects. In 1997 the National Automated Highway Systems Consortium (NAHSC) presented proof of cooperating automated driving on highways with its Free Agent Demonstration which produced viable insights of highway platooning [11].

\section{Spirit of Berlin and MadeInGermany}

"Spirit of Berlin" and "MadeInGermany" are two autonomous cars developed by Freie Universität Berlin (Figure 1).

"Spirit of Berlin" was the participating robot of Team Berlin of Freie Universität Berlin in the DUC [12] and finished as a semifinalists. The vehicle was a retrofitted Dodge
Caravan with drive-by-wire technology, modified so that a handicapped person could drive using a linear lever for brake and gas (the lever controls all intermediate steps between full braking and full acceleration), and a small wheel for steering the front wheels. The rest of the car's components can be controlled through a small contact sensitive panel or using a computer connected to A/D converters.

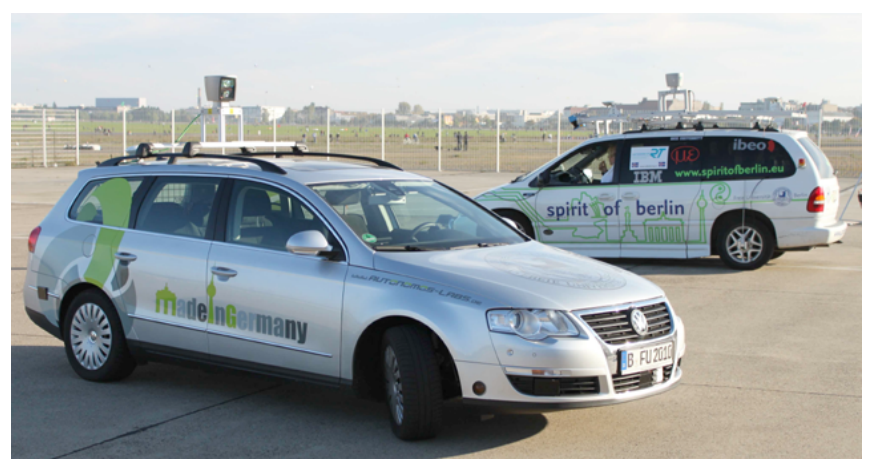

Fig. 1. Our two autonomous cars: "MadeInGermany" (left) and "Spirit of Berlin" (right)

Similarly, we have set up a second autonomous car in 2010 called "MadeInGermany" [13]. "MadeInGermany" is a Volkswagen Passat that has drive-by-wire incorporated at delivery. Thus, actuator commands and sensor information could be sent and retrieved via the CAN protocol.

Six Ibeo Lux laser scanners have been installed in the front and back and an additional Velodyne, 64-layer Lidar Scanner, on top of the roof to sweep the area around the car to detect interfering obstacles. As before we have mounted two cameras for traffic light detection and stereo-vision. All data processing is done by a state-of-the-art mobile computer connected to the CAN-Bus.

We tested our road networks with both vehicles for over several thousand kilometers driving autonomously in urban city scenarios and highway tracks.

\section{Route Network Definitions}

The RDDF was one of the first formats to describe route networks provided by the DARPA which was mainly used for unpaved desert tracks. It consisted of a simple list of longitudes, latitudes, and corridor widths that define the course boundarys, and a list of associated speed limits. Due to its simplicity it is not applicable for urban or highway environments.

For the Urban Challenge the DARPA reworked its RDDF format to a Route Network Definition File (RNDF) format which included specific characterization of urban environment such as intersections, stop sign locations, and parking spots [14]. A RNDF distinguishes between road segments for structured areas such as paved roads defined by a set of waypoints and unstructured areas or 'zones' where free-form navigation within a defined perimeter is allowed.

Discussion of unstructured areas are neglected here for the sake of considering structured areas to discuss their requirements for high-speed motorways. In summary, the 
RNDF combines a list of waypoints to a road lane, where the sequential order of waypoints determine the direction of the lane. Several lanes are then combined to road segments which represents a multi-laned stretch of road. For each waypoint a set of metadata can be applied such as denoting if it is a stop sign, intersection entry or exit, or checkpoint. For a more detailed description of the RNDF format we refer to [14].

The RNDF format proved to be simple yet sufficient modeling language to create road networks on which autonomous missions can be executed as all participants of the DARPA Urban Challenge demonstrated. The Urban Challenge course was only shared between completely autonomous vehicles and trained DARPA operators, such that all participants were aware of this simple road network. Towards introduction of autonomous behavior on real roads alongside conventional human drivers however, a road network definition needs to model all possible aspects of real road networks

Although the RNDF format is simple to understand and sufficient enough to map most road networks, it flaws in several points:

a) only stop signs are defined, missing any other signs and traffic lights

b) roads are modeled as a sequence of waypoints with no interpolation of points in between

c) segments have no real use except for holding lanes together and defining speedlimits

d) lanes can only be connected by "connecting edges" which do not have any properties

e) lanes always have a constant width

f) lanes are not further categorized, e.g. turning lane, parking lane, highway entry and exit

g) lanes have no association with their neighboring lanes, every lane has its own sequence of waypoints

h) left and right lane boundary can only be double yellow, solid yellow, solid white or broken white

i) it is very hard to model traffic islands or roundabouts

j) the RNDF is two-dimensional and does not support bridges, tunnels or overpasses

The list above summarizes most of the features lacking in the RNDF format to model more sophisticated road networks. We will propose some enhancements as RNDFGraph to overcome these flaws in the next section.

It needs to be mentioned, that other road network definitions beside the RNDF format exist, but with a shifted focus on simulation or gaming rather than autonomous driving. The most prominent one would be OpenDRIVE by VIRES Simulationstechnologie $\mathrm{GmbH}$ and the OpenDRIVE community [15] that tries to simplify the data exchange between different driving simulators. It can be combined with OpenCRG to augment the road network with road surface descriptions. OpenDRIVE allows to describe road networks with more data belonging to the road environment than RNDF which also makes it a valid candidate to describe road networks for autonomous driving.

At the time of this work, OpenDRIVE was initially evaluated for use but not readily implemented or tested for autonomous tests. With consideration of legacy work adapted to the RNDF format, we choose to stay with RNDFGraph to be backwards compatible. In addition the OpenDRIVE specification describes a huge amount of additional meta information which is not required for autonomous driving which makes the format bloated for our purposes. However, further evaluation of OpenDRIVE for autonomous driving will certainly be needed as a future task.

\section{RNDFGRAPH}

The RNDFGraph representation is a mapping of the RNDF format into a object graph model with BOOST Graph Library [16] without dropping any information held in the RNDF. The architecture is capable of importing various graph formats such as RNDF or OpenDRIVE in the future while the graph structure itself can be easily serialized into a binary format and thus be loaded from file later.

In the remainder we will describe processing steps while creating the RNDFGraph to gain a more sophisticated road network structure for autonomous path-planning and evaluation. We will describe how RNDFGraph can overcome the RNDF deficiencies described earlier and illustrate optimization techniques to the graph structure itself. Exemplary RNDFGraph structures on various road networks, especially high speed autobahn environments, and results of the optimization process are given in the next section.

\section{A. Graph representation and meta information}

The BOOST Graph is using a directed adjacency list for every edge with a weight based on the edge length. However, the weights can be adjusted in later process steps to differentiate between normal and mirrored lanes. Iterating through the edge list allows the user to make one's way over connecting road segments.

The RNDFGraph defines data maps for every vertex and edge holding static information. It also gives us a convenient access to set dynamic properties of otherwise static objects in our surroundings, e.g. traffic light state.

Thus, a vertex can be augmented with any additional data such as traffic light locations, lane width and stopping restrictions. Edges can be denoted with any additional information such as acceleration or deceleration lane, roundabout or emergency lane.

\section{B. Generating splines using waypoints}

A first and basic processing step is to generate spline representations of the roads given a sequence of waypoints. We choose to model and draw our splines with Akima spline interpolation [17]. The Akima interpolation is a continuously differentiable sub-spline interpolation which resulting spline is less affected by outliers than cubic spline interpolation. Because only data from next neighbor points (in our case 3 ) is used to determine the coefficients of the interpolation polynomial, there is no need to solve large equation systems. Therefore this interpolation method is computationally very efficient.

For road networks this poses several advantages: 
- changing the location of one waypoint does not affect the whole spline

- no oscillations in the road structure

- the resulting graph has a natural visual representation

The splines can be serialized by storing the coefficients of the interpolation polynomial and thus reloaded from files. By working with splines we have a continuous representation of lanes rather than a sequence of waypoints and we have overcome the necessity to assign waypoints to segments. However, waypoint information are not discarded as they still contain vertex information. Because Akima splines tend to make $90^{\circ}$ turns too sharp, we insert a point to make the curve more circular. At the end of any spline another point is added to force a straight ending.

\section{Neighboring and Mirrored Lanes}

Furthermore, given the spline structure representing the lanes, in a subsequent processing step the relationship between lanes can be modeled. Most importantly we need to address the relationship between neighboring lanes and the special case of lanes in driving direction with its oncoming neighboring lanes. To find the left neighbor for every lane a voting system is used. Each lane "votes" how likely every other lane is its left neighbor. With a given threshold the most likely one becomes its left neighbor, this implicates the right neighbor relationship. If two lanes are neighbors and do not have the same direction mirrored edges are added. Mirrored edges share the same start and end vertex as the original but with reversed direction. Using these edges it is possible for the behavior module to plan overtaking maneuvers using oncoming traffic lanes.

\section{Lane Changing Edges}

At this point neighbor relationships are stored with the edges metadata but we can not use standard graph algorithms on our graph since the lanes are not connected with each other yet. To overcome this we add lane changing edges at every start- and checkpoint between a lane and its neighboring edges. At every lane end we add lane changing edges to its neighbors.

\section{E. Connecting Edges}

Connecting edges are the only way to connect one lane to another (or a segment to another). These edges are used at all kind of intersections in urban RNDF. Because each waypoint can have several outgoing connecting edges and the lane gradients at intersections differ very much, connecting edges are not considered as support points for the Akima spline interpolation. As mentioned earlier speed limits are always per segment. To change a speed limit on the autobahn we have to introduce a new segment and connect the lanes of each segment with connecting edges. Since connecting edges do not have any properties the speed limit of the target segment is inherited.

\section{F. Optimizing}

After spline interpolation and lane association, we optimize the graph for wayfinding and real-time decision making. Two major steps are done. First, all waypoints which do not hold any additional information are pruned. This leaves a sparse graph with only essential waypoints such as checkpoints, traffic signs, lane changing vertices, start and end points of lanes. It should be noted, that the graph representation is not affected by the pruning as the lanes are now stored in form of Akima splines. The pruning step is especially useful when modeling non-sparse graphs or a autobahn where many waypoints do not hold additional data.

In a second step an all-pairs-shortest-path (APSP) table is computed for all remaining waypoints. Due to a reduced set of waypoints this should be done after pruning. The APSP-table can then be used by the behavioral executive to calculate shortest path from a given location to any given target mission point. The weights for the APSP table are set accordingly to the length of each edge. Lane changing edges can be penalized with a slightly higher weight and mirrored edges can be punished even harder.

\section{EXPERIMENTS}

We have created over 60 RNDFGraph road networks based on RNDF data. Most route networks are located in Berlin, Germany, which are currently used for local autonomous tests and demonstrations; the others are situated in the US (Houston, Southwest Research Institute, San Antonio, Victorville, etc.) dating back to the DARPA Urban Challenge.

The graphs model real road networks and are aimed to be used for autonomous driving in real traffic which poses extreme requirements on the road representation such as accurate positioning, complete coverage of road signs and traffic lights as well as lane markings and description. Before engaging under real traffic a test scenario on the now disused airport Berlin-Tempelhof (THF) needs to be passed. The artificial road network on the airfield of THF mimics important aspects of the corresponding real road network as a valid test setup. We will present two test setups: one for urban driving in highly inhabited areas with a high number of junctions and one autobahn scenario with requirements for high speed driving.

The urban scenario is located in Berlin-Dahlem, the district where Freie Universität Berlin is located. Figure 2 depicts the road network of Dahlem with two close-ups of junction points and figure 3 shows the corresponding prerequisite road network THF-Dahlem on the THF airfield. THF-Dahlem has a course length of about $13.3 \mathrm{~km}$ where as Dahlem is about twice as long which may not be immediately apparent in the figure due to different scales.

Both road networks were provided in a RNDF format and then converted into RNDFGraph with the optimization steps described above. The RNDFGraph structure enables easier path-planning and more accurate low-level controlling of the autonomous car when using it in real-time. The following Table I shows attributes of the road network before and after optimization to RNDFGraph and the optimization duration. 


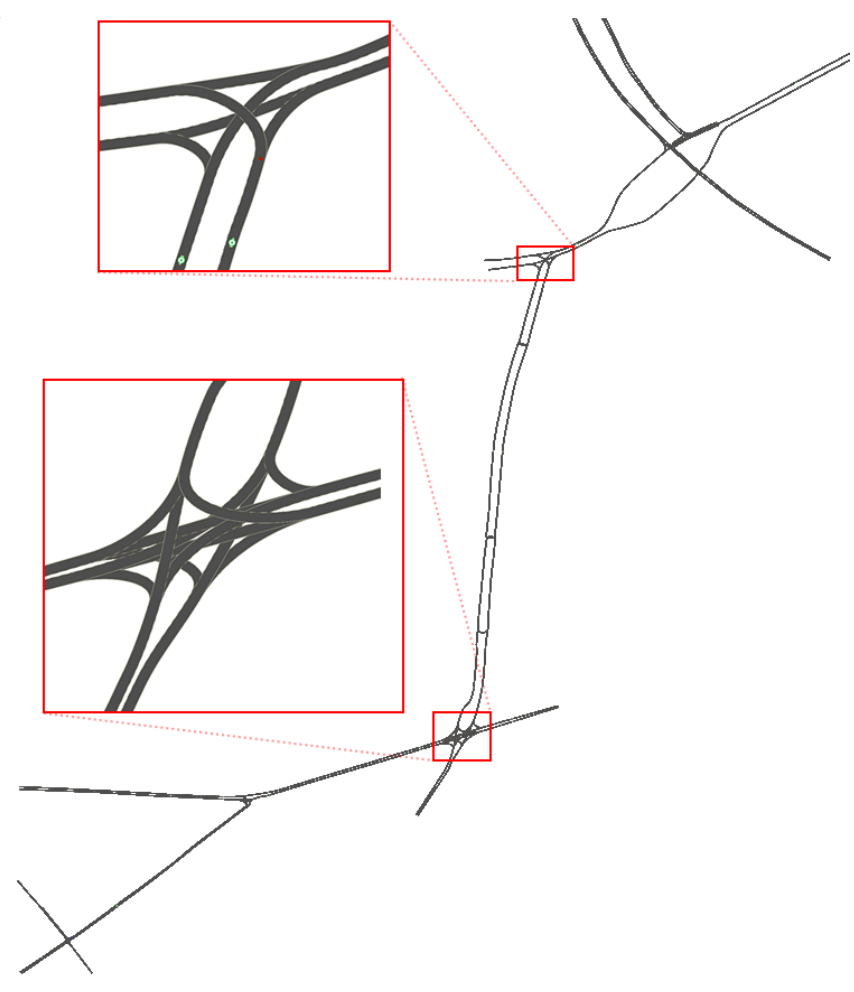

Fig. 2. Dahlem road network

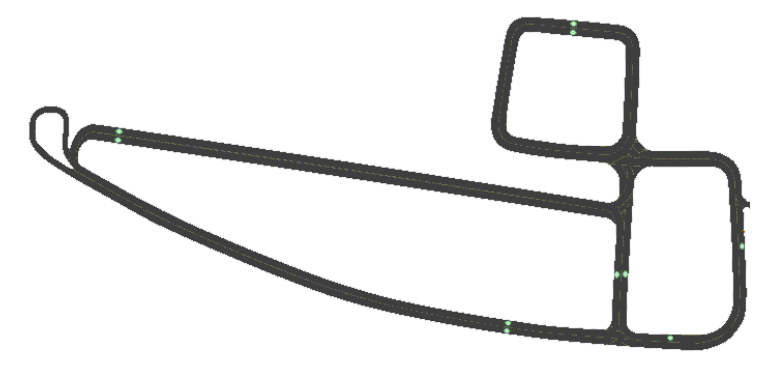

Fig. 3. THF-Dahlem road network, two junctions have been magnified as a close-up

\begin{tabular}{|l|c|c|}
\hline & THF-Dahlem & Dahlem \\
\hline total route network length (km) & 13.358 & 26.718 \\
\hline vertex count before optimization & 109 & 614 \\
\hline vertex count after optimization & 128 & 296 \\
\hline edge count before optimization & 124 & 626 \\
\hline edge count after optimization & 159 & 301 \\
\hline time to prune vertices (ms) & 0.1320 & 1.2266 \\
\hline time to build mirrored graph (ms) & 0.0926 & 0.4508 \\
\hline time to add lane changing edges (ms) & 0.7786 & 4.5997 \\
\hline
\end{tabular}

TABLE I

DAHLEM ROAD NETWORK BEFORE AND AFTER OPTIMIZATION
Both vertex and edge count were greatly reduced in Dahlem to $48 \%$ with mostly interim vertices between intersections pruned. The same does not hold for the artificially created THF-Dahlem route network as the lanes between intersections have been designed much shorter for demonstration purposes. Instead, vertex and edge count increased slightly due to added lane changing edges. The total optimization time is below $7 \mathrm{~ms}$ and thus not critical.

For our high speed tests we have chosen the AutomobilVerkehrs- und Übungs-Straße (AVUS) in the south-western district of Berlin. AVUS was used as a motor racing circuit in the past, while now it became part of Autobahn 115 (A 115), one of Germany's most used motorways. We choose to use a part of AVUS for autonomous highway driving to evaluate the limits of our path-planning and low-level controller system. Figure 4 depicts the segment of AVUS and and a close-up of a highway junction. To prepare for a AVUS test drive we have created a speedway THF-AVUS on the THF airfield (Figure 5). The AVUS network is about 10 times longer than THF-AVUS (74.2 km vs. $7.5 \mathrm{~km}$ )

\begin{tabular}{|l|c|c|}
\hline & THF-AVUS & AVUS \\
\hline total route network length $(\mathrm{km})$ & 7.528 & 74.285 \\
\hline vertex count before optimization & 72 & 578 \\
\hline vertex count after optimization & 81 & 214 \\
\hline edge count before optimization & 78 & 573 \\
\hline edge count after optimization & 84 & 229 \\
\hline time to prune vertices (ms) & 0.1377 & 0.9833 \\
\hline time to build mirrored graph $(\mathrm{ms})$ & 0.1096 & 0.2801 \\
\hline time to add lane changing edges $(\mathrm{ms})$ & 0.5765 & 5.4019 \\
\hline
\end{tabular}

TABLE II

AVUS ROAD NETWORK BEFORE AND AFTER OPTIMIZATION

The results in Table II are similar to Dahlem. Vertex and edge count slightly increased for THF-AVUS and were reduced to $37 \%$ and $40 \%$ for AVUS. The increased reduction can be explained by the long straight passages on the autobahn where vertices do not hold additional information. The total optimization time here was below $7 \mathrm{~ms}$.

\section{CONCLUSION}

This work has presented some enhancements for route networks defined as RNDFGraph to be used as a digital map for autonomous vehicles. The RNDFGraph overcomes various flaws of the underlying RNDF structure. In comparison to the flaws we have mapped out in Section III, we can draw following conclusion: With the usage of additional properties set to vertices and edges we resolved points a) and e), f), and h). Our spline representation solves problem b) and c). With special consideration on neighboring, lane changing, and connecting edges we address item d) and g). No improvement have been made for the remaining points i) and j), and will be of major importance for future work and research.

The RNDFGraph provides a road network with additional information for autonomous behavior such as continuous spline sampling, lane relationships and lane change information, to be used by subsequent path-planning and low-level 


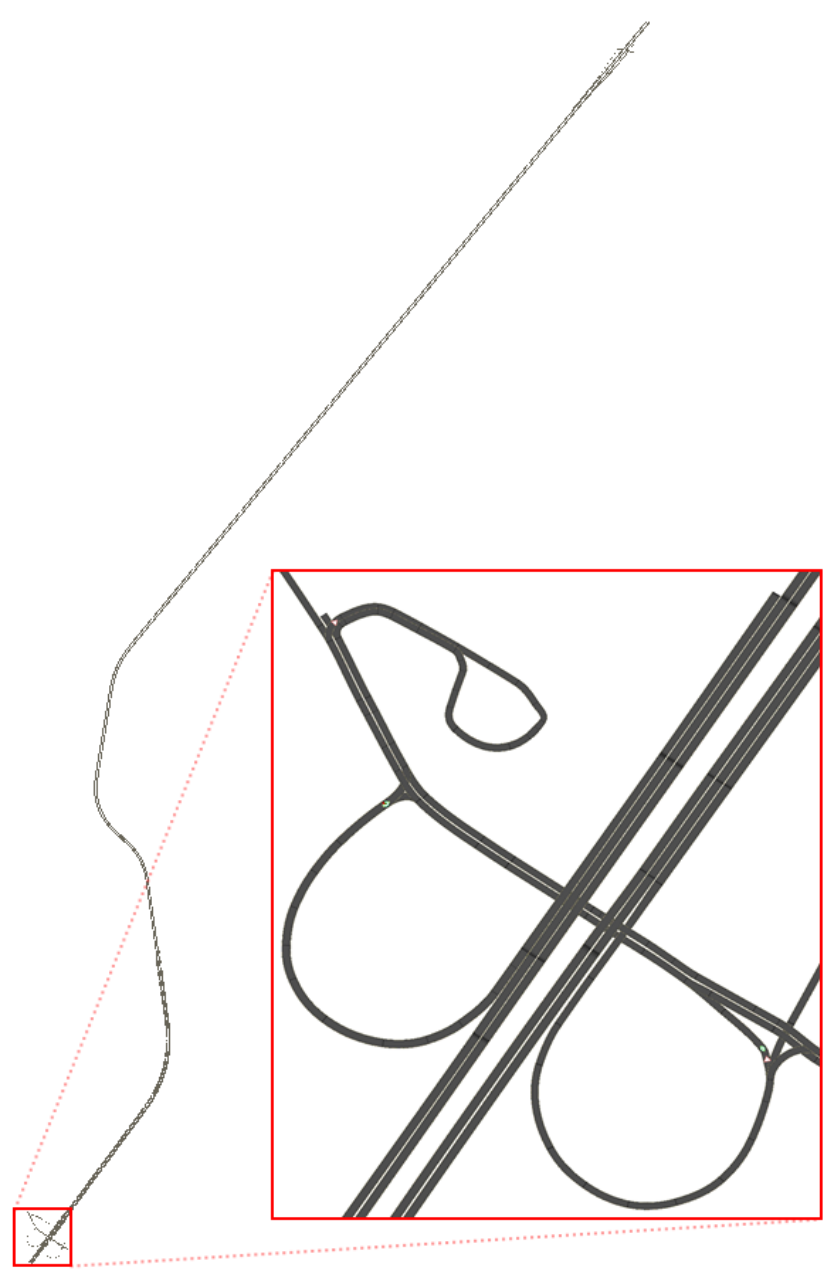

Fig. 4. AVUS road network (left) and a close up of a highway junction (bottom, right)

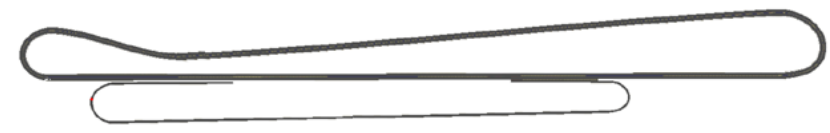

Fig. 5. Speedway THF-AVUS road network

controller modules. Special interest has been put on highway scenarios on high-speed German autobahn where multiple autonomous test runs have taken place in real traffic. An optimization step is able to reduce vertex and edge count for non-sparse route networks while optimization time can be neglected. Results of the optimization and several route networks have been presented.

\section{ACKNOWLEDGMENTS}

This work was supported by the German Federal Ministry of Education and Research (BMBF), ForMaT Initiative as InnoLab AutoNOMOS (FKZ 03FO2022). We would also like to thank the anonymous reviewers for their feedback.

\section{REFERENCES}

[1] U. Özgüner, C. Stiller, and K. Redmill, "Systems for safety and autonomous behavior in cars: The DARPA Grand Challenge experience," Proceedings of the IEEE, vol. 95, pp. 397-412, Feb 2007.

[2] M. Buehler, K. Iagnemma, and S. Singh, The 2005 DARPA Grand Challenge: The Great Robot Race. Springer Tracts In Advanced Robotics, 1 ed., 2006. ISBN-13: 978-3540734284.

[3] M. Buehler, K. Iagnemma, and S. Singh, "Special Issue: Special Issue on the 2007 DARPA Urban Challenge, Part I-III," Journal of Field Robotics, vol. 25, pp. 423 - 860, August 2008.

[4] C. Urmson, J. Anhalt, M. Clark, T. Galatali, J. P. Gonzalez, J. Gowdy, A. Gutierrez, S. Harbaugh, M. Johnson-Roberson, H. Kato, P. Koon, K. Peterson, B. Smith, S. Spiker, E. Tryzelaar, and W. Whittaker, "High speed navigation of unrehearsed terrain: Red team technology for grand challenge 2004," Tech. Rep. CMU-RI-TR-04-37, Carnegie Mellon University, June 2004.

[5] S. Thrun, M. Montemerlo, H. Dahlkamp, D. Stavens, A. Aron, J. Diebel, P. Fong, J. Gale, M. Halpenny, G. Hoffmann, K. Lau, C. Oakley, M. Palatucci, V. Pratt, P. Stang, S. Strohband, C. Dupont, L.-E. Jendrossek, C. Koelen, C. Markey, C. Rummel, J. van Niekerk, E. Jensen, P. Alessandrini, G. Bradski, B. Davies, S. Ettinger, A. Kaehler, A. Nefian, and P. Mahoney, "Stanley: The robot that won the darpa grand challenge," Journal of Field Robotics, vol. 23, pp. 661 - 692, September 2006.

[6] C. Urmson, J. Anhalt, D. Bagnell, C. Baker, R. Bittner, M. N. Clark, J. Dolan, D. Duggins, T. Galatali, C. Geyer, M. Gittleman, S. Harbaugh, M. Hebert, T. M. Howard, S. Kolski, A. Kelly, M. Likhachev, M. McNaughton, N. Miller, K. Peterson, B. Pilnick, R. Rajkumar, P. Rybski, B. Salesky, Y.-W. Seo, S. Singh, J. Snider, A. Stentz, W. Whittaker, Z. Wolkowicki, J. Ziglar, H. Bae, T. Brown, D. Demitrish, B. Litkouhi, J. Nickolaou, V. Sadekar, W. Zhang, J. Struble, M. Taylor, M. Darms, and D. Ferguson, "Autonomous driving in urban environments: Boss and the urban challenge," Journal of Field Robotics, vol. 25, pp. 425 - 466, July 2008.

[7] B. Ulmer, "VITA - An Autonomous Road Vehicle (ARV) for Collision Avoidance in Traffic," Proceedings of the Intelligent Vehicles '92 Symposium., pp. 36 - 41, June 1992. ISBN: 0-7803-0747-X.

[8] M. Maurer, R. Behringer, S. Fürst, F. Thomanek, and E. D. Dickmanns, "A compact vision system for road vehicle guidance," Proceedings of the 13th International Conference on Pattern Recognition, 1996., vol. 3, pp. 313 - 317, August 1996. ISBN: 0-8186-7282-X.

[9] C. Thorpe, M. H. Hebert, T. Kanade, and S. A. Shafer, "Vision and navigation for the Carnegie-Mellon Navlab," IEEE Transactions on Pattern Analysis and Machine Intelligence, vol. 10, pp. 362 - 372, May 1988. ISSN: 0162-8828.

[10] A. Hattori, A. Hosaka, and M. Taniguchi, "Driving control system for an autonomous vehicle using multiple observed point information," Proceedings of the Intelligent Vehicles '92 Symposium., pp. 207 212, June 1992. ISBN: 0-7803-0747-X.

[11] C. Thorpe, T. Jochem, and D. Pomerleau, "The 1997 automated highway free agent demonstration," IEEE Conference on Intelligent Transportation Systems, November, 1997, pp. 496 - 501., pp. 496 501, November 1997.

[12] J. Rojo, R. Rojas, K. Gunnarsson, M. Simon, F. Wiesel, F. Ruff, L. Wolter, F. Zilly, N. Santrac, T. Ganjineh, A. Sarkohi, F. Ulbrich, D. Latotzky, B. Jankovic, G. Hohl, T. Wisspeintner, S. May, K. Pervoelz, W. Nowak, F. Maurelli, and D. Droeschel, "Spirit of Berlin: An autonomous car for the DARPA Urban Challenge - hardware and software architecture," tech. rep., Freie Universität Berlin, June 2007.

[13] B. Fischer, T. Ganjineh, D. Göhring, S. Hempel, T. Langner, D. Latotzky, A. Reuschenbach, M. Schnürmacher, E. Tapia, F. Ulbrich, V. Schauermann, M. Wang, F. Wiesel, T. Zoppke, and R. Rojas, “Technical report AutoNOMOS 2010," tech. rep., Freie Universität Berlin, January 2011

[14] DARPA Urban Challenge, "Route network definition file (RNDF) and mission data file (MDF) formats," tech. rep., Defense Advanced Research Projects Agency, March 2007.

[15] M. Dupuis, OpenDRIVE Format Specification. VIRES Simulationstechnologie $\mathrm{GmbH}, 2010$.

[16] J. G. Siek, L.-Q. Lee, and A. Lumsdaine, The Boost Graph Library: User Guide and Reference Manual. Addison-Wesley Professional, December 2001. ISBN-13: 978-0201729146.

[17] H. Akima, "A new method of interpolation and smooth curve fitting based on local procedures," Journal of ACM, vol. 17, no. 4, pp. 589$602,1970$. 